Article

\title{
5-ALA Fluorescence Image Guided Resection of Glioblastoma Multiforme: A Meta-Analysis of the Literature
}

\section{Samy Eljamel}

Neurological Surgery, High Tech Neuro \& Micro Surgery, Edinburgh EH3 8JB, UK;

E-Mail: professer.ms.eljamel@gmail.com

Academic Editor: Michael R. Hamblin

Received: 26 January 2015 / Accepted: 27 April 2015 / Published: 7 May 2015

\begin{abstract}
Background: Glioblastoma multiforme (GBM) is one of the most deadly cancers in humans. Despite recent advances in anti-cancer therapies, most patients with GBM die from local disease progression. Fluorescence image guided surgical resection (FIGR) was recently advocated to enhance local control of GBM. This is meta-analyses of 5-aminolevulinic (5-ALA) induced FIGR. Materials: Review of the literature produced 503 potential publications; only 20 of these fulfilled the inclusion criteria of this analysis, including a total of 565 patients treated with 5-ALA-FIGR reporting on its outcomes and 800 histological samples reporting 5-ALA-FIGR sensitivity and specificity. Results: The mean gross total resection (GTR) rate was 75.4\% (95\% CI: 67.4-83.5, $p<0.001)$. The mean time to tumor progression (TTP) was 8.1 months (95\% CI: 4.7-12, $p<0.001)$. The mean overall survival gain reported was 6.2 months $(95 \% \mathrm{CI}:-1-13, p<0.001)$. The specificity was $88.9 \%$ (95\% CI: 83.9-93.9, $p<0.001$ ) and the sensitivity was $82.6 \%$ (95\% CI: 73.9-91.9, $p<0.001$ ). Conclusion: 5-ALA-FIGR in GBM is highly sensitive and specific, and imparts significant benefits to patients in terms of improved GTR and TTP.
\end{abstract}

Keywords: ALA; FIGS; FIGR; glioma; glioblastoma; brain tumor; surgery

\section{Introduction}

Glioblastoma multiforme (GBM) is a locally invasive malignant brain cancer that evaded and eluded cure because of its resistance to current surgical, chemotherapeutic and radiotherapeutic techniques. The best that can be offered to patients today is 14 months median survival with maximum safe surgical resection and concurrent chemoradiotherapy using Temozolomide and external beam 
radiotherapy [1]. To date, almost all patients have died from local brain recurrence and more than $80 \%$ of recurrences developed within $2 \mathrm{~cm}$ of the resection margin. Hence, the reason for failure of current therapeutic techniques seems more likely than not due to their failure to impart radical local disease control $[2,3]$.

One of the most innovative techniques that came to the forefront in recent years to improve the local control rate in GBM surgery was the use of 5-aminolevulinic acid (5-ALA)-induced fluorescence to guide surgery (FIGR). 5-ALA induces fluorescence due to accumulation of protoporphyrin IX (PpIX) in the cells of GBM leading to intraoperative biological tagging of these cells, the operating surgeon can see, seek and resect easily without increasing collateral brain damage [3-26]. A randomized controlled trial (RCT) demonstrated FIGR had doubled the number of patients who had total resection of the enhancing component of their GBM and significantly prolonged their tumor-free survival [5]. FIGR was approved in the European Union and its use expanded worldwide. It was envisaged that FIGR would undergo the same stages of implementation as any other newly introduced surgical technique. Initially there would be two camps: the skeptics who would not use the technology because of doubts mainly self-doubt about their ability to adopt and use the technology effectively and fear that they will loose some business if they cannot adopt the new technology. The second groups of surgeons includes those who are eager to adopt new technology to improve their work and takes it to the next level and drum some new business. The skeptics will use every opportunity to criticize any presentation or publication and introduce hurdles and excuses to slow down adoption of the new technology. Once most surgeons adopt a new technology, there would be an initial precaution to use it selectively because of the steep learning curve. Once they are comfortable with the new technology, there would be enthusiasm to use the technology widely, leading to less selectivity and expansion of indications in the grey area. As a result of this wider usage surgeons start to experience unwanted outcomes, which leads to refining the indications for using the new technology and the introduction of stringent selection of patients. FIGR has been in use for over two decades, hence it is time to perform meta-analyses of the published literature to find out if the initial results of FIGR had been replicated and whether FIGR achieves the desired outcomes when widely adopted by surgeons outside the stringent criteria and scrutiny of an RCT protocol.

\section{Results}

Out of a potentially relevant 26 studies, five reported the use of fluorescein-induced fluorescence image guided resection and one reported on mTHPC (Foscan, Biolitec, Dublin, Ireland)-induced fluorescence image guided surgery (Figure 1). Therefore, only 20 fulfilled all inclusion criteria for this meta-analysis. One study by Eljamel et al. 2008 [15] had Photoferin repetitive photodynamic therapy (rPDT) in addition to 5-ALA-FIGR, it was included in the gross total resection (GTR) part of the analysis as rPDT would not had an effect on GTR. The rest of outcome measures it the analyses were performed with this study in and out for completeness as it would not be possible to discern whether 5-ALA-FIGR or rPDT had the major effect. Eight studies were used in meta-analyses of sensitivity and specificity and 12 were used in meta-analyses of FIGR-outcomes. 


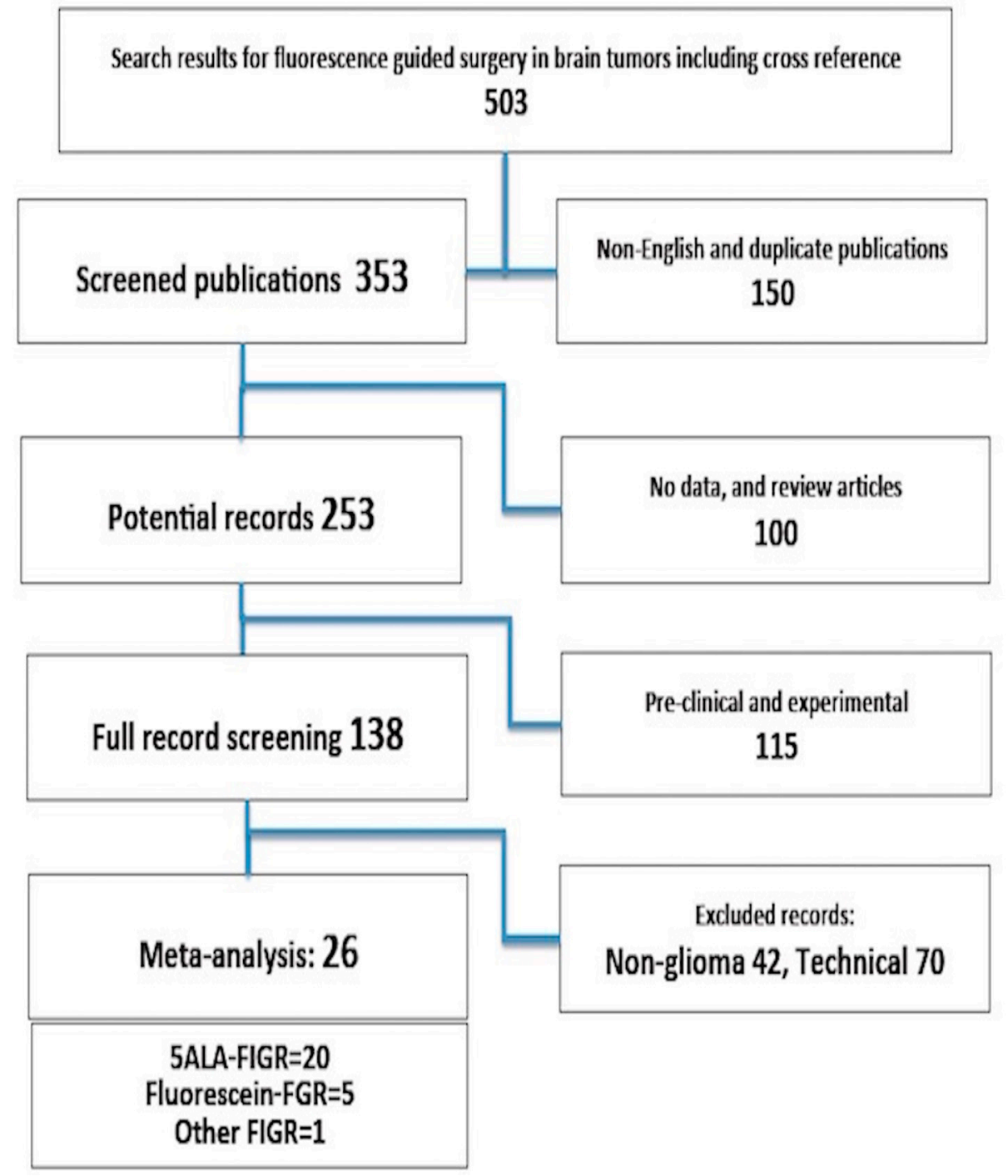

Figure 1. Details of the literature search.

\subsection{5-Aminolevulinic Acid (5-ALA)-Induced Fluorescence to Guide Surgery (FIGR) Gross Total} Resection (GTR)

GTR was defined in most studies as absence of contrast enhancement in the resection region of GBM on post-operative T1-weighted MRI scan obtained within the first $72 \mathrm{~h}$ after surgery. However, at least one study [11] defined GTR as resection of more than $98 \%$ of the enhanced lesion. Eleven studies have reported clearly this outcome; 418/565 (75.4\%) had GTR in these studies (95\% CI: 67.4-83.5, $p<0.001)$ Figure 2. 


\begin{tabular}{lccc}
\multicolumn{2}{l}{ Heterogeneity } & & \\
tau^2 & $Q(\mathrm{df}=10)$ & Het. p-Value & $I^{\wedge} 2$ \\
0.014 & 51.185 & $<0.001$ & 80.463
\end{tabular}

Forest Plot

\begin{tabular}{llll} 
Studies & \multicolumn{2}{c}{ Estimate $(95$ c.I. $)$} & Ev/Trt \\
& & & \\
Stummer et al 2008 & 0.667 & $(0.587,0.746)$ & $90 / 135$ \\
Piquer et al 2014 & 0.767 & $(0.615,0.918)$ & $23 / 30$ \\
Stummer et al 2000 & 0.635 & $(0.504,0.765)$ & $33 / 52$ \\
Della Puppa et al 2014 & 0.926 & $(0.872,0.979)$ & $87 / 94$ \\
Pastor et al 2013 & 0.676 & $(0.519,0.834)$ & $23 / 34$ \\
Diez Valle et al 2013 & 0.680 & $(0.589,0.771)$ & $68 / 100$ \\
Della Puppa et al 2 2013 & 0.742 & $(0.588,0.896)$ & $23 / 31$ \\
Jaquesson et al 2013 & 0.682 & $(0.487,0.876)$ & $15 / 22$ \\
Diez Valle et al 2 2011 & 0.833 & $(0.712,0.955)$ & $30 / 36$ \\
Feigl et al 2010 & 0.889 & $(0.744,1.000)$ & $16 / 18$ \\
Eljamel et al 2008 & 0.769 & $(0.540,0.998)$ & $10 / 13$ \\
& & & \\
Overall (I^2=80\%, P<0.001) & 0.754 & $(0.674,0.835)$ & $418 / 565$
\end{tabular}

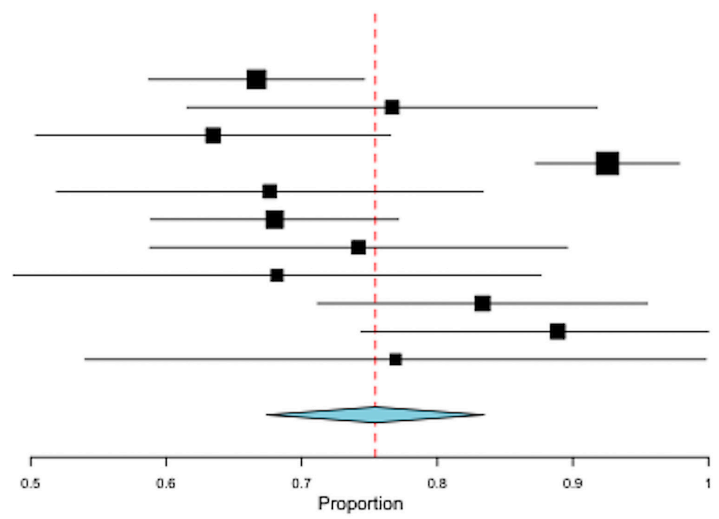

Figure 2. Meta-analysis of gross total resection (GTR) in glioblastoma multiforme (GBM) using 5-aminolevulinic fluorescence image guided surgical resection (5-ALA-FIGR). (Squares represent the mean, the bigger the square is the more weight given because of the narrower $95 \% \mathrm{CI}$, the diamond represent the mean in the combined data in the meta-analyses.).

\subsection{5-ALA-FIGR Time to Tumor Progression}

Time to tumor progression (TTP) should always be considered the primary endpoint of any study of GBM treatment. Three studies reported TTP. One of these studies reported 5-ALA-FIGR and rPDT, therefore the analyses were performed with this study-data in and out. Despite heterogeneity, which was significant $(p<0.05)$, the mean TTP of the collated data was 8 months (95\% CI: 4.3-12 months, $p<0.001)$, Figure 3 .
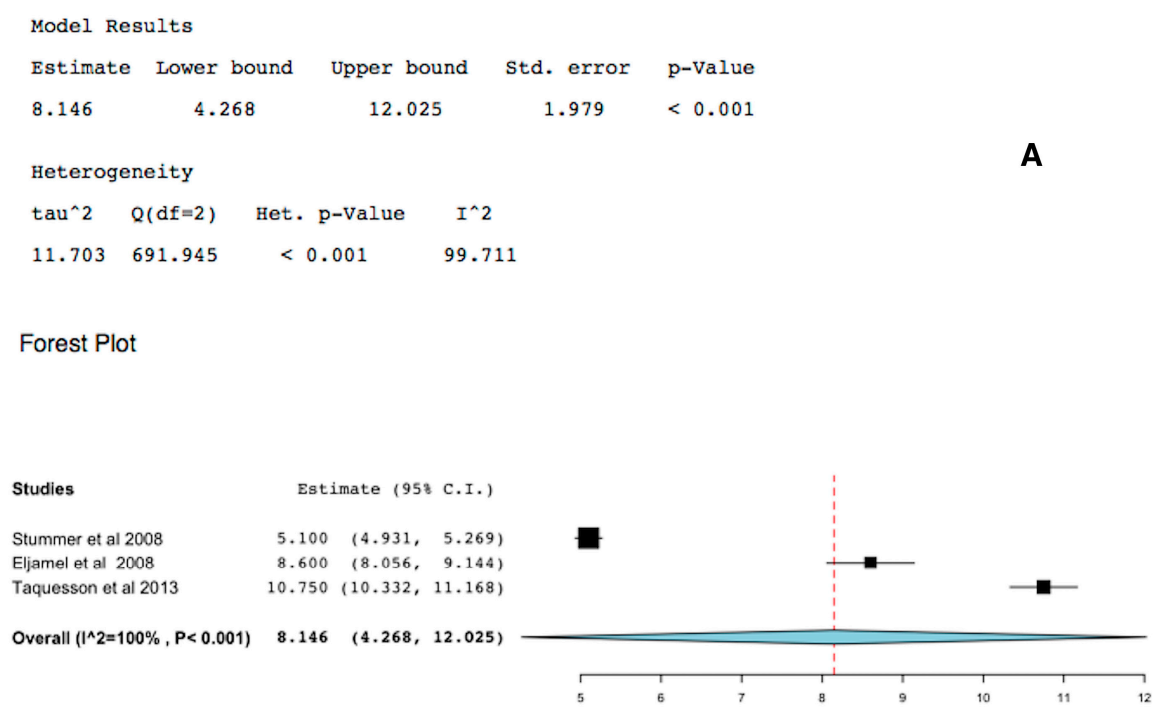

Figure 3. Cont. 


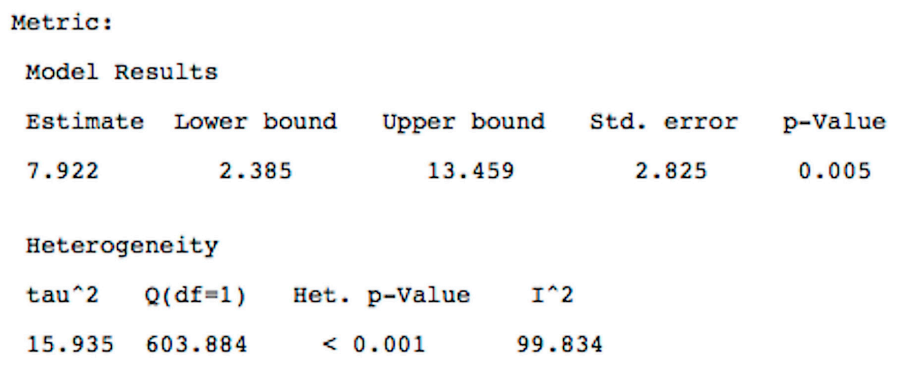

\section{B}

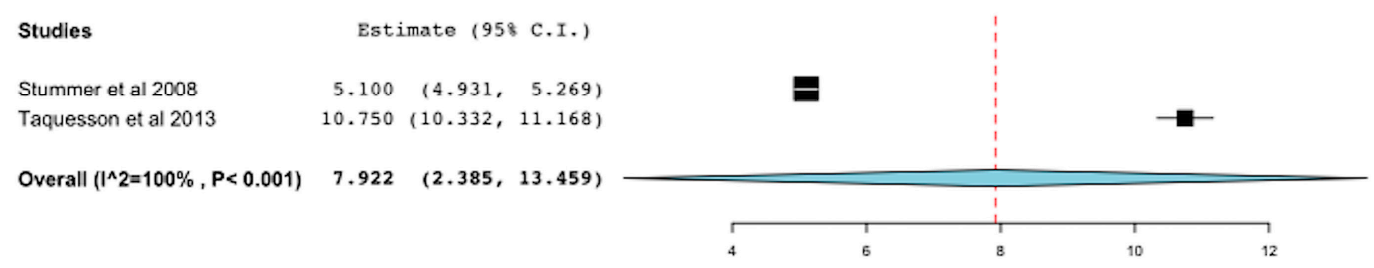

Figure 3. Forest plot (meta-analysis) and heterogeneity of 5-ALA-FIGR time to tumor progression (TTP). (A) excludes study [15] and (B) includes it, because study [15] had repetitive PDT (rPDT) in addition to 5-ALA-FIGR. (Squares represent the mean, the bigger the square is the more weight given because of the narrower $95 \% \mathrm{CI}$, the diamond represent the mean in the combined data in the meta-analyses.).

\subsection{5-ALA-FIGR Survival}

Over all survival in GBM is an inappropriate primary endpoint because patients and surgeons found it difficult to control what other treatments and interventions are carried out after tumor recurrence. Nevertheless, three studies with matched controls (two were RCTs) reported on overall survival of GBM patients treated with 5-ALA-FIGR or not. One of these studies was compounded by the fact that rPDT was used in addition to 5-ALA-FIGR in the study arm [15]; therefore, the analyses were performed both including and excluding this study. The difference in survival in favor of 5-ALA-FIGR was $4-6.2$ months (95\% CI: -1 to $13, p<0.001)$, Figure 4 . However, the $95 \%$ CI was very wide and the effect may well be due to adjuvant therapies, therefore 5-ALA-FIGR alone does not seem to impart significant overall survival benefit on its own or the current studies were underpowered to detect a difference between the study arms. 


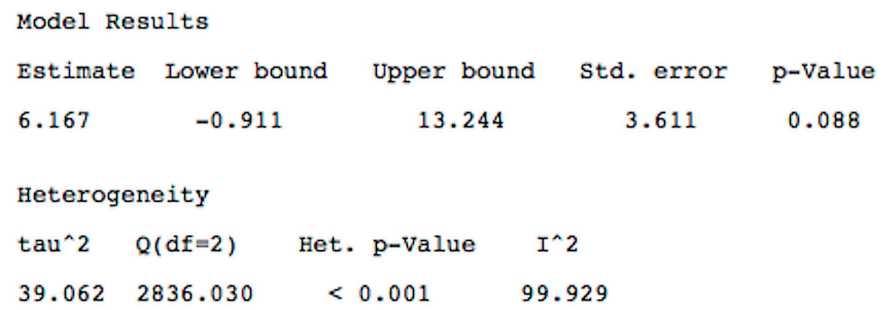

Forest Plot

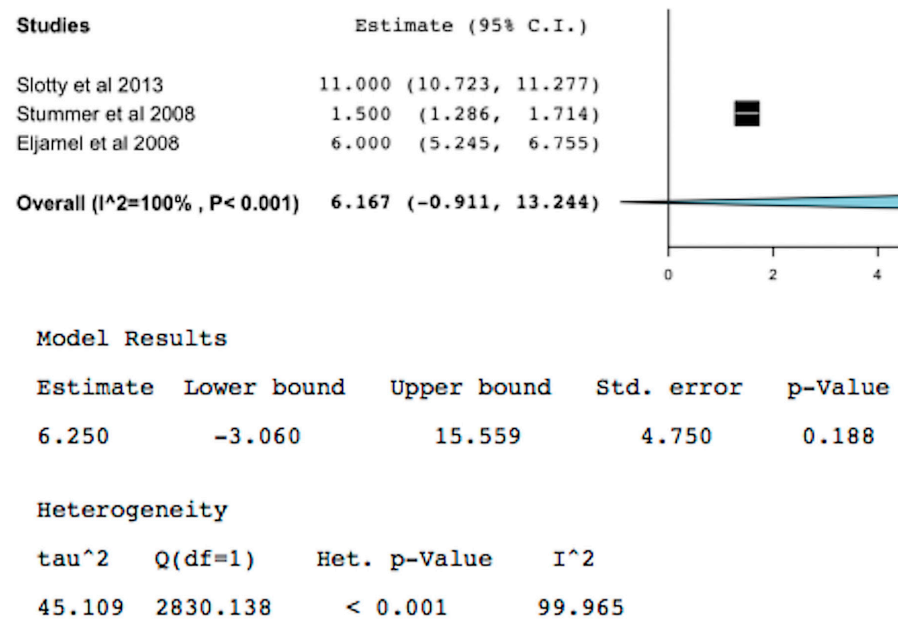

Model Results

Estimate Lower bound Upper bound std. error p-Value

$\begin{array}{lllll}6.250 & -3.060 & 15.559 & 4.750 & 0.188\end{array}$

Heterogeneity

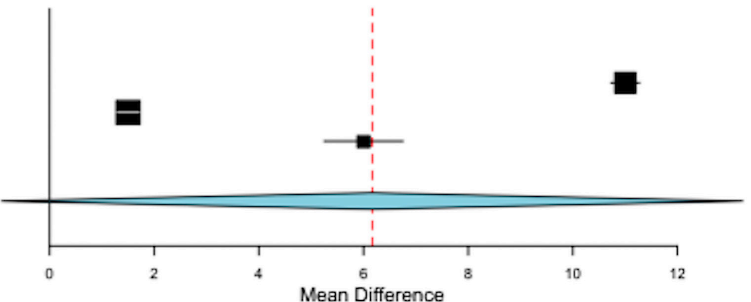

Forest Plot

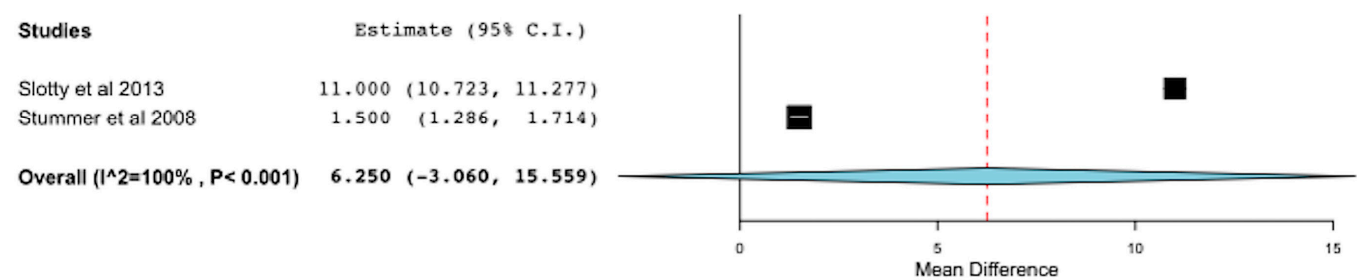

Figure 4. Forest plot (meta-analysis) and heterogeneity of 5-ALA-FIGR survival difference. (A) excludes study [15] and (B) includes it, because study [15] had rPDT in addition to 5-ALA-FIGR. (Squares represent the mean, the bigger the square is the more weight given because of the narrower $95 \% \mathrm{CI}$, the diamond represent the mean in the combined data in the meta-analyses.).

\subsection{5-ALA-FIGR Specificity}

Specificity was defined as the percent of specimens that were fluorescence-negative and contained no GBM cells. Eight studies reported 5-ALA-FIGR specificity including 800 histopathological specimens. Two studies included non-GBM data and therefore the analyses were performed with and without these studies. The specificity was $88.8 \%-90 \%$ (95\% CI: 83.8\%-93.9\%, $p<0.001)$, Figure 5. 

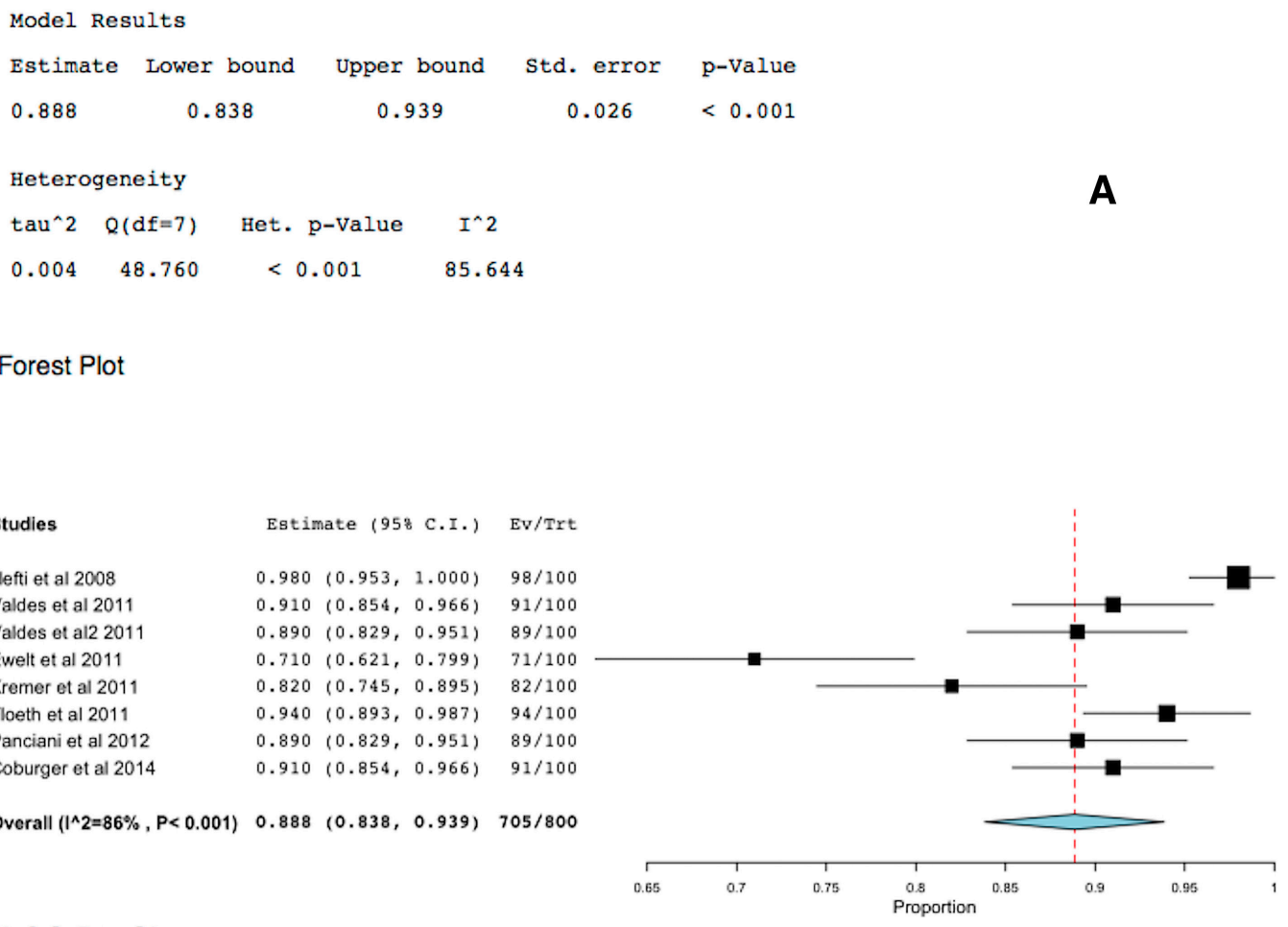

Model Results

Estimate Lower bound Upper bound Std. error p-Value

$\begin{array}{llll}0.900 & 0.871 & 0.929 & 0.015<0.001\end{array}$

Heterogeneity

tau^2 $Q(\mathrm{df}=5) \quad$ Het. p-Value $I^{\wedge} 2$

$\begin{array}{llll}0.000 & 7.560 & 0.182 & 33.859\end{array}$

Forest Plot
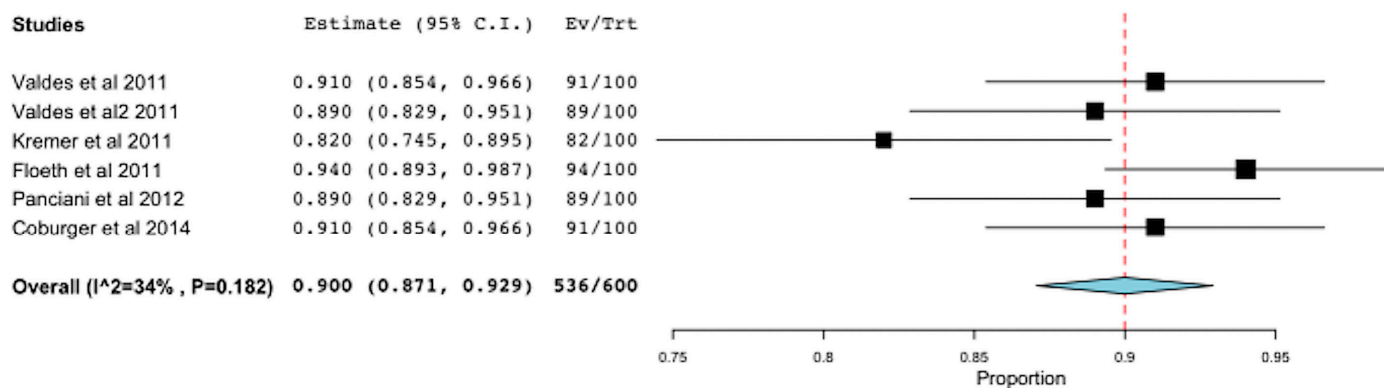

Figure 5. Forest plot (meta-analysis) and heterogeneity of 5-ALA-FIGR specificity. (A) excludes studies with non-GBM data and (B) includes them, because studies included other types of tumors other than GBM. (Squares represent the mean, the bigger the square is the more weight given because of the narrower $95 \% \mathrm{CI}$, the diamond represent the mean in the combined data in the meta-analyses.).

\subsection{5-ALA-FIGR Sensitivity}

Sensitivity was defined as the percent of specimens of GBM that fluoresced using 5-ALA-FIGR. Eight studies reported on this. Two studies included non-GBM data and therefore the analyses were 
performed with and without these studies. The sensitivity of 5-ALA-FIGR was $81 \%-82.6 \%$ (95\% CI: $73.9 \%-91.4 \%, p<0.001)$, Figure 6.

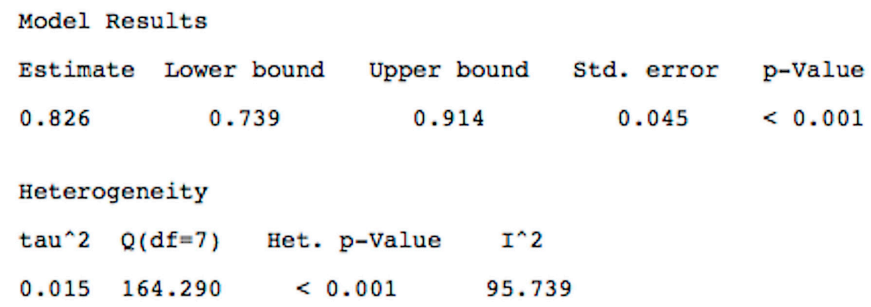

Forest Plot

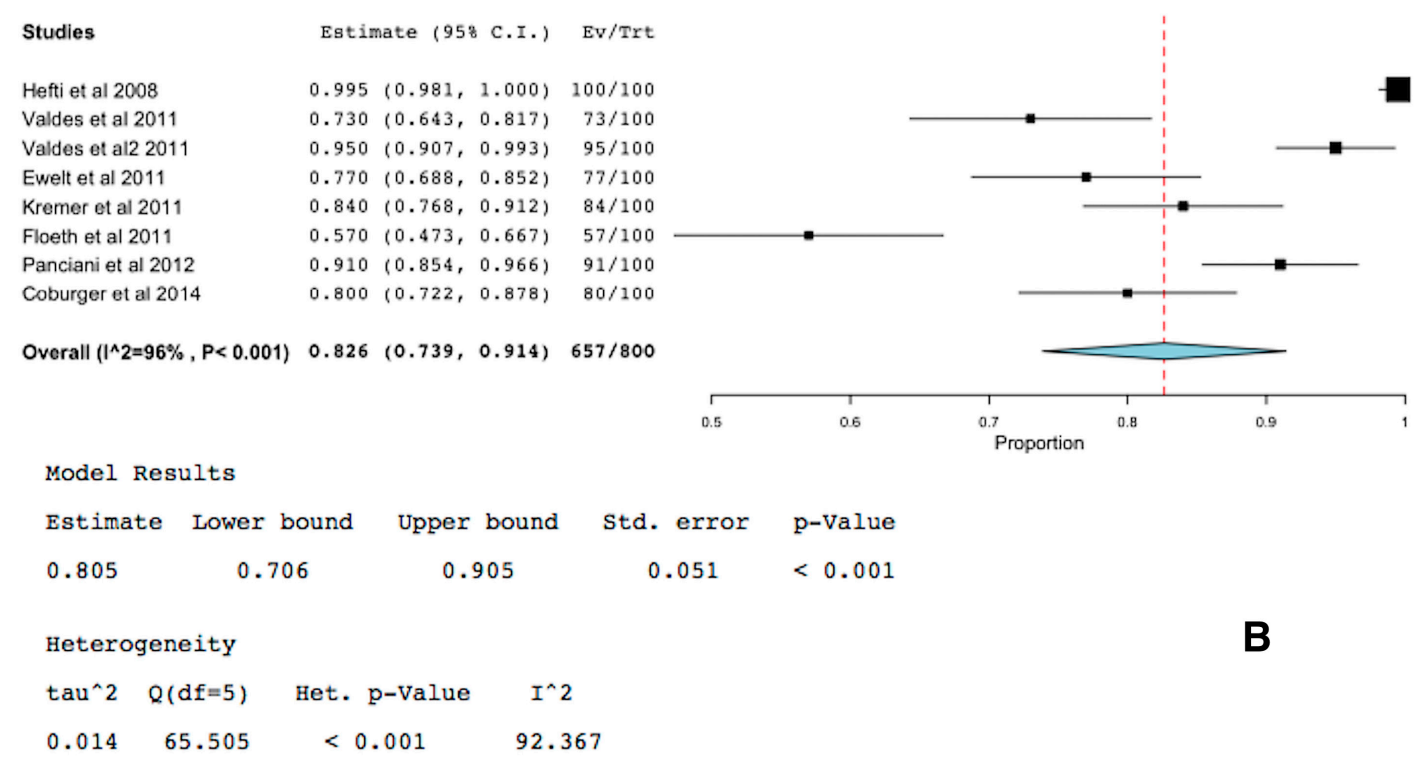

Forest Plot

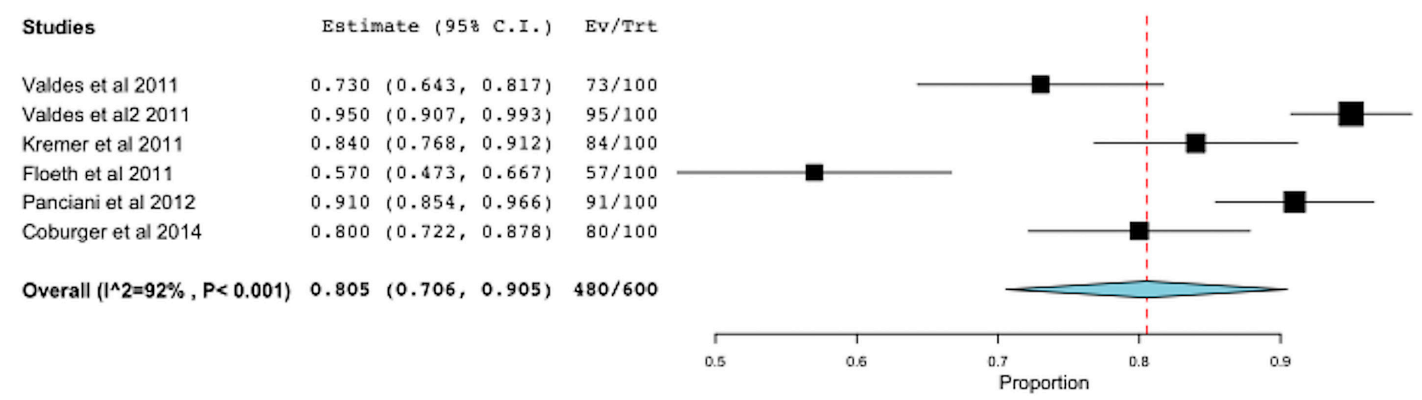

Figure 6. Forest plot (meta-analysis) and heterogeneity of 5-ALA-FIGR sensitivity. (A) excludes studies with non-GBM data and (B) includes them, because studies [19,22] included other types of tumors other than GBM. (Squares represent the mean, the bigger the square is the more weight given because of the narrower 95\% CI, the diamond represent the mean in the combined data in the meta-analyses.). 


\section{Discussion}

GTR is an independent prognostic factor for TTP and survival in GBM and a GTR of 98\% or more of the enhancing lesion on MRI is considered to be best for GBM surgery [2,3,7]. In the best hands, GTR under white light microsurgery is achieved in about $36 \%$ of patients [5] and under the care of non-specialized neurosurgeons more likely to be about 25\%. 5-ALA-FIGR improved the GTR in GBM to $>64 \%$ [2-6] and when compared to white light microsurgery 5-ALA-FIGR was highly significant $(p<0.001)$ [5]. In this meta-analyses that included 11 studies that clearly reported this outcome [4,5,7-15]; 418/565 (75.4\%) had GTR in these studies (95\% CI: 67.4-83.5, $p<0.001)$. Furthermore, it is likely that the extent of surgical resection is much more than post operative MRI scan revealed because several studies had demonstrated that 5-ALA-fluorescence revealed and enabled resection of a much wider lesion than the enhanced MRI scan had revealed, it is estimated that fluorescent GBM extends by $10 \mathrm{~mm}$ or more beyond the enhanced edge [16,17]. Therefore, postoperative enhanced MRI scan within the first $72 \mathrm{~h}$ is a poor tool to evaluate the true extent of surgical resection in GBM. However, it is the standard of care currently in use.

Time to tumor progression (TTP) should always be considered the primary endpoint of any study evaluating GBM treatment, because in the context of an RCT, it would be difficult and probably un-ethical to control what further therapy or intervention the patient, surgeon or oncologist will choose after GBM recurrences. Three studies included in this meta-analysis reported TTP [5,12,15]. Despite the heterogeneity of these studies, which was significant $(p<0.05)$, the mean TTP with 5-ALA-FIGR was 8.1 months $(95 \% \mathrm{CI}: 4.3-12$ months, $p<0.001)$. In the two RTCs included in this study, the TTP was significantly longer in the 5-ALA-FIGR arm compared to white microsurgery arm $(p<0.001)[5,15]$.

Though one of these studies was compounded by the addition of Photoferin rPDT [15], repeating the analysis without this study, the TTP was still significant in favor of 5-ALA-FIGR.

Furthermore, patients in the 5-ALA-FIGR had significantly less rescue interventions, such as further surgery, chemotherapy and other interventions [5], resulting in significant healthcare cost-savings and longer quality of life for patients.

Overall survival in GBM is an inappropriate primary endpoint because patients and surgeons found it difficult to control what other treatments and interventions are carried out after tumor recurrence. Nevertheless, three studies with matched controls (two were RTCs) reported on overall survival of GBM patients treated with 5-ALA-FIGR or not $[5,15,18]$. The difference in survival in favor of 5-ALA-FIGR was 6.2 months $(95 \% \mathrm{CI}:-1$ to $13, p<0.001)$. However, the $95 \%$ confidence interval was very wide and heterogeneity was also significant because of the aforementioned reasons: patients in the control group often received further surgery and rescue therapies that diluted the survival gain of patients who received 5-ALA-FIGR upfront. Though one of these studies was compounded by the addition of Photoferin rPDT [15], repeating the analysis without this study the outcome was still significant in favor of 5-ALA-FIGR. However, because these studies were underpowered and there was no control for rescue and adjuvant therapies, no firm conclusions can be drawn from the published data so far to indicate 5-ALA-FIGR alone imparts significant over all survival advantage.

The current meta-analyses based on eight studies that reported histopathological confirmation of over 800 samples of 5-ALA-fluorescence positive or negative [19-26] demonstrated 5-ALA-FIGR had very high specificity of $88.8 \%(95 \%$ CI: $83.8 \%-93.9 \%, p<0.001)$ and equally high sensitivity of 
82.6\% (95\% CI: $73.9 \%-91.4 \%, p<0.001)$. Excluding two studies that were not pure GBMs did not change materially these results. This high sensitivity and specificity of 5-ALA-FIGR is unmatched by any other intraoperative adjuvants used to enhance the GTR such as image guided surgery (ISG), intraoperative ultrasound (IoUS) or intraoperative MRI (iMRI).

In a previous systematic review of the literature that included 12 studies reported sensitivity of fluorescence guided surgery (FGS) for high-grade glioma of $84 \%$ and specificity of $91 \%$ and reduced the risk of tumor progression (HR 0.73) [27]. However, the aforementioned review included studies using fluorescein-guided surgery, which were excluded from this meta-analyses [28-32] to eliminate heterogeneity, as the mechanism by which fluorescein and 5-ALA mark GBM cells is entirely different. We have also excluded a further matched controlled study of FIGR, which was not included in the previous systematic review because it reported the use of mTHPC (Foscan) to guide surgery [33]. Furthermore, another systematic review of the literature published two years ago included only 10 studies and concluded ALA-fluorescence guided surgery had a sensitivity of $87 \%$, specificity of $89 \%$ [34], which are higher than this meta-analysis because of the stricter selection criteria and this meta-analyses had additional published data. The selective biases that occur as new technology is adopted into main-steam practice are evident by the slide downward of these results as the selection of suitable patients becomes blurred as the experience develops during the learning curve.

It is clear that the extent of surgical resection is an important significant prognostic factor in the TTP and survival in high-grade gliomas and 5-ALA-FIGR is the best available technique to achieve maximum safe surgical resection, which is associated with very few local or systemic side effects [5,35,36]. RCT [5] demonstrated similar complication rates in the 5-ALA-FIGR and the control group. However, the holly grail of eradicating the GBM by extending the surgical resection should not override the basic principle of patient safety and preservation of function as extending surgical resection from $>98 \%$ excision to total removal of the enhancing lesion resulted in similar outcomes. Furthermore, tumors near eloquent brain areas may require additional techniques such as awake craniotomy to minimize the risk of collateral damage.

\section{Experimental Section}

The medical literature was searched extensively, beginning with basic searches of the MEDLINE/PubMed service of the US National Library of Medicine, using the MeSH (medical subject heading) terms "fluorescence," "5-aminolevulinic acid (5-ALA)," "glioma," "brain," "glioblastoma," "neurosurgery," and "surgery" in various combinations. Furthermore, Web of Knowledge database, BIOSIS Previews, Cochrane library, and Web of Science were searched.

Each article of interest was screened and its reference list was checked to make sure that no relevant article was missed. The Internet itself was searched for leads to articles appearing in journals not indexed in these databases.

The relevant clinical studies were selected on the basis of one of the following selection criteria:

(1) Reported sensitivity and specificity of 5-ALA-fluorescence identification of GBM;

(2) Gross total resection of 5-ALA-fluorescence-image guided resection (FIGR) was reported;

(3) The outcomes in terms of time to tumor progression or overall survival were reported. 
When the same patient population was used in several publications from the same authors or same institutions, only the most recent study was included in this meta-analysis. Full-text copies of the articles upon which this meta-analysis was based were examined Articles that did not deal with 5-ALA-guided surgery, and non-GBM diagnosis were excluded. Articles that were not in English, contained insufficient data or were technical in nature were also excluded. Only one study was in French was included as it was possible to translate it into English [12].

Eventually, 503 potential articles were accumulated for review (Figure 1). Only 20 out of a potential 26 articles that met the inclusion criteria, were included in the final meta-analyses.

The meta-analyses were conducted according to statistical heterogeneity between the studies using Open MetaAnalyst Software version 0.1 (Brown University, Providence, RI, USA) for Mac. If there was no heterogeneity, fixed effects model was used for meta-analysis; otherwise, a random effect model was used. Statistical heterogeneity was explored by $\chi 2$ and inconsistency (I2) statistics; an I2 value of $50 \%$ or more represented substantial heterogeneity. Meta-analysis is a statistical technique for combining the findings of two or more independent studies to provide a more precise estimates of treatment effects, giving due weight to the size of the different studies included in the analyses.

\section{Conclusions}

5-ALA-FIGR in GBM is highly sensitive and specific, and imparts significant benefits to patients in terms of improved GTR and TTP. Although the extent of surgical resection is an important prognostic factor in GBMs and 5-ALA-FIGR significantly improved the extent of surgical resection of GBM, at the moment it is not clear that 5-ALA-FIGR alone improves overall survival of GBM. Further RCT with large study sample and appropriate control of adjuvant therapies is warranted.

\section{Acknowledgments}

Samy Eljamel acknowledges the contribution of his students and the staff in the Scottish PDT Center for the support during the collection of these data over the years and is grateful for the authors who published their data with enough information to enable this analysis.

\section{Author Contributions}

Samy Eljamel had carried out the analyses, drafting and reviewing the manuscript and critically reviewed the studies upon which this analysis was based.

\section{Conflicts of Interest}

The authors declare no conflict of interest. 


\section{References}

1. Stupp, R.; Hegi, M.E.; Mason, W.P.; van den Bent, M.J.; Taphoorn, M.J.B.; Janzer, R.C.; Ludwin, S.K.; Allgeier, A.; Fisher, B.; Belanger, K.; et al. Effects of radiotherapy with concomitant and adjuvant temozolomide versus radiotherapy alone on survival in glioblastoma in a randomized phase III study: 5-year analysis of the EORTC-NCIC trial. Lancet Oncol. 2009, 10, 459-466.

2. Eljamel, M.S. Brain photodiagnosis (PD), fluorescence guided resection (FGR) and photodynamic therapy (PDT): Past, present and future. Photodiagn. Photodyn. Ther. 2008, 5, 29-35.

3. Eljamel, S. Photodynamic applications in brain tumors: A comprehensive review of the literature. Photodiagn. Photodyn. Ther. 2010, 7, 76-85.

4. Stummer, W.; Novotny, A.; Stepp, H.; Goetz, C.; Bise, K.; Reulen, H.J. Fluorescence-guided resection of glioblastoma multiforme by using 5-aminolevulinic acid-induced porphyrins: A prospective study in 52 consecutive patients. J. Neurosurg. 2000, 93, 1003-1013.

5. Stummer, W.; Pichlmeier, U.; Meinel, T.; Wiestler, O.D.; Zanella, F.; Reulen, H.J.; ALA-Glioma Study Group. Fluorescence-guided surgery with 5-aminolevulinic acid for resection of malignant glioma: A randomised controlled multicentre phase III trial. Lancet Oncol. 2006, 7, 392-401.

6. Eljamel, M.S. Fluorescence image-guided surgery of brain tumors: Explained step-by-step. Photodiagn. Photodyn. Ther. 2008, 5, 260-263.

7. Della Puppa, A.; Ciccarino, P.; Lombardi, G.; Rolma, G.; Cecchin, D.; Rossetto, M. 5-Aminolevulinic acid fluorescence in high grade glioma surgery: Surgical outcome, intraoperative findings, and fluorescence patterns. Biomed. Res. Int. 2014, 2014, 232561.

8. Piquer, J.; Llácer, J.L.; Rovira, V.; Riesgo, P.; Rodriguez, R.; Cremades, A. Fluorescence-guided surgery and biopsy in gliomas with an exoscope system. Biomed. Res. Int. 2014, 2014, 207974.

9. Pastor, J.; Vega-Zelaya, L.; Pulido, P.; Garnés-Camarena, O.; Abreu, A.; Sola, R.G. Role of intraoperative neurophysiological monitoring during fluorescence-guided resection surgery. Acta Neurochir. (Wien) 2013, 155, 2201-2213.

10. Díez Valle, R.; Slof, J.; Galván, J.; Arza, C.; Romariz, C.; Vidal, C. VISIONA study researchers. Observational, retrospective study of the effectiveness of 5-aminolevulinic acid in malignant glioma surgery in Spain (The VISIONA study). Neurologia 2014, 29, 131-138.

11. Della Puppa, A.; de Pellegrin, S.; d'Avella, E.; Gioffrè, G.; Rossetto, M.; Gerardi, A.; Lombardi, G.; Manara, R.; Munari, M.; Saladini, M.; et al. 5-aminolevulinic acid (5-ALA) fluorescence guided surgery of high-grade gliomas in eloquent areas assisted by functional mapping. Our experience and review of the literature. Acta Neurochir. (Wien) 2013, 155, 965-972.

12. Jacquesson, T.; Ducray, F.; Maucort-Boulch, D.; Armoiry, X.; Louis-Tisserand, G.; Mbaye, M.; Pelissou-Guyotat, I.; Guyotat, J. Surgery of high-grade gliomas guided by fluorescence: A retrospective study of 22 patients. Neurochirurgie 2013, 59, 9-16.

13. Díez Valle, R.; Tejada Solis, S.; Idoate Gastearena, M.A.; García de Eulate, R.; Domínguez Echávarri, P.; Aristu Mendiroz, J. Surgery guided by 5-aminolevulinic fluorescence in glioblastoma: Volumetric analysis of extent of resection in single-center experience. J. Neurooncol. 2011, 102, 105-113. 
14. Feigl, G.C.; Ritz, R.; Moraes, M.; Klein, J.; Ramina, K.; Gharabaghi, A.; Krischek, B.; Danz, S.; Bornemann, A.; Liebsch, M.; et al. Resection of malignant brain tumors in eloquent cortical areas: A new multimodal approach combining 5-aminolevulinic acid and intraoperative monitoring. J. Neurosurg. 2010, 113, 352-357.

15. Eljamel, M.S.; Goodman, C.; Moseley, H. ALA and Photofrin fluorescence-guided resection and repetitive PDT in glioblastoma multiforme: A single centre Phase III randomised controlled trial. Lasers Med. Sci. 2008, 23, 361-367.

16. Eljamel, S.; Petersen, M.; Valentine, R.; Buist, R.; Goodman, C.; Moseley, H.; Eljamel, S. Comparison of intraoperative fluorescence and MRI image guided neuronavigation in malignant brain tumours, a prospective controlled study. Photodiagn. Photodyn. Ther. 2013, 10, 356-361.

17. Roberts, D.W.; Valdés, P.A.; Harris, B.T.; Fontaine, K.M.; Hartov, A.; Fan, X.; Ji, S.; Lollis, S.S.; Pogue, B.W.; Leblond, F.; et al. Coregistered fluorescence-enhanced tumor resection of malignant glioma: Relationships between $\delta$-aminolevulinic acid-induced protoporphyrin IX fluorescence, magnetic resonance imaging enhancement, and neuropathological parameters. J. Neurosurg. 2011, 114, 595-603.

18. Slotty, P.J.; Siantidis, B.; Beez, T.; Steiger, H.J.; Sabel, M. The impact of improved treatment strategies on overall survival in glioblastoma patients. Acta Neurochir. (Wien) 2013, 155, 959-963.

19. Hefti, M.; von Campe, G.; Moschopulos, M.; Siegner, A.; Looser, H.; Landolt, H. 5-aminolevulinic acid induced protoporphyrin IX fluorescence in high-grade glioma surgery: A one-year experience at a single institutuion. Swiss Med. Wkly. 2008, 138, 180-185.

20. Valdés, P.A.; Leblond, F.; Kim, A.; Harris, B.T.; Wilson, B.C.; Fan, X.; Tosteson, T.D.; Hartov, A.; Ji, S.; Erkmen, K.; et al. Quantitative fluorescence in intracranial tumor: Implications for ALA-induced PpIX as an intraoperative biomarker. J. Neurosurg. 2011, 115, 11-17.

21. Valdés, P.A.; Kim, A.; Leblond, F.; Conde, O.M.; Harris, B.T.; Paulsen, K.D.; Wilson, B.C.; Roberts, D.W. Combined fluorescence and reflectance spectroscopy for in vivo quantification of cancer biomarkers in low- and high-grade glioma surgery. J. Biomed. Opt. 2011, 16, 116007.

22. Ewelt, C.; Floeth, F.W.; Felsberg, J.; Steiger, H.J.; Sabel, M.; Langen, K.J.; Stoffels, G.; Stummer, W. Finding the anaplastic focus in diffuse gliomas: The value of Gd-DTPA enhanced MRI, FET-PET, and intraoperative, ALA-derived tissue fluorescence. Clin. Neurol. Neurosurg. 2011, 113, 541-547.

23. Kremer, P.; Fardanesh, M.; Ding, R.; Pritsch, M.; Zoubaa, S.; Frei, E. Intraoperative fluorescence staining of malignant brain tumors using 5-aminofluorescein-labeled albumin. Neurosurgery 2009, 64, ons53-ons61.

24. Floeth, F.W.; Sabel, M.; Ewelt, C.; Stummer, W.; Felsberg, J.; Reifenberger, G.; Steiger, H.J.; Stoffels, G.; Coenen, H.H.; Langen, K.J. Comparison of ${ }^{18}$ F-FET PET and 5-ALA fluorescence in cerebral gliomas. Eur. J. Nucl. Med. Mol. Imaging 2011, 38, 731-741.

25. Panciani, P.P.; Fontanella, M.; Schatlo, B.; Garbossa, D.; Agnoletti, A.; Ducati, A.; Lanotte, M. Fluorescence and image guided resection in high grade glioma. Clin. Neurol. Neurosurg. 2012, $114,37-41$. 
26. Coburger, J.; Engelke, J.; Scheuerle, A.; Thal, D.R.; Hlavac, M.; Wirtz, C.R.; König, R. Tumor detection with 5-aminolevulinic acid fluorescence and Gd-DTPA-enhanced intraoperative MRI at the border of contrast-enhancing lesions: A prospective study based on histopathological assessment. Neurosurg. Focus 2014, 36, E3.

27. Su, X.; Huang, Q.F.; Chen, H.L.; Chen, J. Fluorescence-guided resection of high-grade gliomas: A systematic review and meta-analysis. Photodiagn. Photodyn. Ther. 2014, 11, 451-458.

28. Acerbi, F.; Broggi, M.; Eoli, M.; Anghileri, E.; Cavallo, C.; Boffano, C.; Cordella, R.; Cuppini, L.; Pollo, B.; Schiariti, M.; et al. Is fluorescein-guided technique able to help in resection of high-grade gliomas? Neurosurg. Focus 2014, 36, E5.

29. Acerbi, F.; Broggi, M.; Eoli, M.; Anghileri, E.; Cuppini, L.; Pollo, B.; Schiariti, M.; Visintini, S.; Orsi, C.; Franzini, A.; et al. Fluorescein-guided surgery for grade IV gliomas with a dedicated filter on the surgical microscope: Preliminary results in 12 cases. Acta Neurochir. (Wien) 2013, $155,1277-1286$.

30. Chen, B.; Wang, H.; Ge, P.; Zhao, J.; Li, W.; Gu, H.; Wang, G.; Luo, Y.; Chen, D. Gross total resection of glioma with the intraoperative fluorescence-guidance of fluorescein sodium. Int. J. Med. Sci. 2012, 9, 708-714.

31. Koc, K.; Anik, I.; Cabuk, B.; Ceylan, S. Fluorescein sodium-guided surgery in glioblastoma multiforme: A prospective evaluation. Br. J. Neurosurg. 2008, 22, 99-103.

32. Shinoda, J.; Yano, H.; Yoshimura, S.; Okumura, A.; Kaku, Y.; Iwama, T.; Sakai, N. Fluorescence-guided resection of glioblastoma multiforme by using high-dose fluorescein sodium. Technical note. J. Neurosurg. 2003, 99, 597-603.

33. Zimmermann, A.; Ritsch-Marte, M.; Kostron, H. mTHPC-mediated photodynamic diagnosis of malignant brain tumours. Photochem. Photobiol. 2001, 74, 611-616.

34. Zhao, S.; Wu, J.; Wang, C.; Liu, H.; Dong, X.; Shi, C.; Shi, C.; Liu, Y.; Teng, L.; Han, D.; et al. Intraoperative fluorescence-guided resection of high-grade malignant gliomas using 5-aminolevulinic acid-induced porphyrins: A systematic review and meta-analysis of prospective studies. PLoS ONE 2013, 28, e63682.

35. Chung, I.W.; Eljamel, S. Risk factors for developing oral 5-aminolevulinic acid-induced side effects in patients undergoing fluorescence guided resection. Photodiagn. Photodyn. Ther. 2013, 10, 362-367.

36. Stummer, W.; Tonn, J.C.; Mehdorn, H.M.; Nestler, U.; Franz, K.; Goetz, C.; Bink, A.; Pichlmeier, U.; ALA-Glioma Study Group. Counterbalancing risks and gains from extended resections in malignant glioma surgery: A supplemental analysis from the randomized 5-aminolevulinic acid glioma resection study. Clinical study. J. Neurosurg. 2011, 114, 613-623.

(C) 2015 by the authors; licensee MDPI, Basel, Switzerland. This article is an open access article distributed under the terms and conditions of the Creative Commons Attribution license (http://creativecommons.org/licenses/by/4.0/). 